\title{
Inhalt / Sommaire
}

Erwin H. Ackerknecht, Jean Starobinski medical historian 211

Bronislaw Baczko, Le Cercle et la spirale 213

Erwin H. Ackerknecht, Some remarks concerning bureaucracy and medicine 221

François Azouvi, Homo duplex 229

Isaac Benguigui, Charles-Gaspard de la Rive (1770-1834), médecin aliéniste et physicien 245

Urs Boschung, Albrecht von Haller und der praktische Arzt seiner Zeit 253

Marguerite Carozzi, Bonnet, Spallanzani, and Voltaire on Regeneration of Heads in Snails: a Continuation of the Spontaneous Generation Debate 265

$\begin{array}{lr}\text { Jean Jacques Dreifuss, Moritz Schiff et la vivisection } & 289\end{array}$

Charles Hahn, Fondements historiques du principe de la reconstruction
en chirurgie vasculaire

Huldrych M. Koelbing, Remarques à propos de l'évolution des connaissances sur le cerveau 315

$\begin{array}{ll}\text { Philippe Mudry, Médecins et spécialistes } & 329\end{array}$

Jackie Pigeaud, Homo quadratus. Variations sur la beauté et la santé dans la médecine antique

Marie-Louise Portmann, Der Basler Arzt Theodor Zwinger III (1658-1724) und seine Arbeit über ein langes Leben

Olivier Rieppel, The dream of Charles Bonnet (1720-1793) 359

Jacques Roger, Lamarck et Jean-Jacques Rousseau 369

Gerhard Rudolph, Amor medicabilis herbis. Un essai au XVIII siècle de se guérir de l'érotomanie par la Ciguë

B. Rüttimann, Souvenirs des guerres napoléoniennes: points de vue des blessés et de leụr médecins

Francis Schiller, The Mystique of the Frontal Lobes 415

Michael Shepherd, Sozialgeschichte: die Geschichte eines Begriffs 425

Antoinette Stettler, Geschichtliches über den Schmerz in Pathologie,

Nervenphysiologie und Mentalität

Owsei Temkin, Hippocrates as the Physician of Democritus 455

Jean Théodoridès, Le personnage du médecin dans l'œuvre romanesque de Stendhal 465

Antoine Thivel, Diagnostic et pronostic à l'époque d'Hippocrate et à la nôtre 479

Dora B. Weiner, Philippe Pinel, linguist: his work as translator and editor 499

Buchbesprechungen

Albert von Rohr, Die Medizinische Poliklinik der Universität Zürich 1835 bis 1983

(Erwin H. Ackerknecht)

Liselotte Kuntner, Die Gebärhaltung der Frau (Erwin H. Ackerknecht) 511

Blas Bruni Celli, Bibliografía Hipocrática (Félix Bloch)

Maria Teresa Monti, Catalogo del Fondo Haller della Biblioteca Nazionale Braidense di Milano (Urs Boschung)

Friedrich Tiedemann, Das Hirn des Negers mit dem des Europäers und Orang-Outangs verglichen (Urs Boschung) 
Fridolf Kudlien, Ärzte im Nationalsozialismus (Carl Haffter) 516

C. A.Meier, Der Traum als Medizin (Carl Haffter) 517

Willem F.Daems, Johann Anton Grass (Carl Haffter) 518

Michler/Benedum, Einführung in die Medizinische Fachsprache (Heinz Balmer) 518

Ortrun Riha, Meister Alexanders Monatsregeln (Hans-Rudolf Fehlmann) 519

Johannes Krähe, Die Diskussion um den ärztlichen Kunstfehler (Antoinette Stettler) 521

Huldrych M. Koelbing, Christian Sigismund Fingers Dissertation «Über den schädlichen Einfluß von Furcht und Schreck bei der Pest» (Antoinette Stettler) 522

Heinrich Schipperges, Historische Konzepte einer Theoretischen Pathologie (Antoinette Stettler)

Paul Feyerabend, Christian Thomas (Hrsg.), Kunst und Wissenschaft (Antoinette Stettler)

Rio Howard, La bibliothèque et le laboratoire de Guy de la Brosse au Jardin des Plantes à Paris (Roger Mayer)

W.Gerlach, Otto Hahn. Ein Forscherleben (G.Schramm)

Jochen Kreidel, Johann Heinrich Dierbach (G.Schramm) 526

Alfred Stückelberger, Vestigia Democritea (Jean Druey) 527

Christoph Meinel, In physicis futurum saeculum respicio (Jean Druey) 529

Konrad Moll, Der junge Leibniz (Karl von Meyenn) 530

Dederick C. Ward und Albert V.Carozzi, Geology Emerging (Oliver Rieppel) 532

Alireza Djafari Naini, Geschichte der Zahlentheorie im Orient, im Mittelalter
und zu Beginn der Neuzeit (Erwin Neuenschwander)

Andor Kertész, Georg Cantor, Schöpfer der Mengenlehre (Erwin Neuenschwander) 533

Zum Werk Leonhard Eulers (Erwin Neuenschwander) 534

Judith Bryce, Cosimo Bartoli. The Career of a Florentine Polymath (Antoinette Stettler) 535

$\begin{array}{ll}\text { Mitteilungen } & 537\end{array}$ 